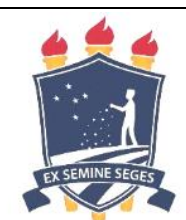

\title{
Uso de metodologia ativa na formação de médicos veterinários residentes para atuação no Sistema Único de Saúde: potencialidades e fragilidades
}

\author{
[Use of active methodology in the training of veterinary residents to work in the Unified Health \\ System: potentialities and fragilities]
}

"Artigo Científico/Scientific Article"

\author{
Jéssica Cristianne Mazer Bernardi ${ }^{1, *}$, Diogo Diógenes Medeiros Diniz ${ }^{1}$, \\ Marcelo Ribeiro dos Santos ${ }^{1}$, Yuri Mateus Lima de Albuquerque ${ }^{2}$, \\ Daniel Friguglietti Brandespim ${ }^{1}$
}

\author{
${ }^{1}$ Departamento de Medicina Veterinária, Universidade Federal Rural do Pernambuco, Recife- PE, Brasil. \\ ${ }^{2}$ Programa de Pós-Graduação em Biociência Animal, Departamento de Morfologia e Fisiologia Animal, Universidade \\ Federal Rural do Pernambuco, Recife- PE, Brasil. \\ *Autor para correspondência/Corresponding author: E-mail: jessicacmbernardi@ hotmail.com
}

\begin{abstract}
Resumo
O Programa de Residência em Área Profissional da Saúde em Medicina Veterinária da Universidade Federal Rural do Pernambuco tem como parte do objetivo capacitar residentes Médicos Veterinários por meio do treinamento em serviço para atuação na saúde pública, nas áreas de vigilância em saúde. Dessa forma, o presente estudo objetivou descrever o método utilizado na formação teórica e prática dos residentes, previamente à inserção dos mesmos no Sistema Único de Saúde (SUS), destacando suas potencialidades e fragilidades. Antes do início das atividades na Secretaria de Vigilância em Saúde e no Núcleo de Apoio à Saúde da Família, foram oferecidas duas disciplinas utilizando-se metodologias ativas: Políticas Públicas em Saúde e Integração Ensino Serviço Comunidade. Ao final de cada disciplina foi solicitado aos residentes aprovados (100\% dos residentes matriculados) que relatassem suas opiniões sobre as mesmas, elencando as potencialidades e as fragilidades. As potencialidades mais relatadas foram: uso de metodologias ativas como Aprendizagem Baseada em Problemas (ABP) e estudos de casos (77,8\%), leitura de artigos e elaboração de resumos $(27,8 \%)$ e esclarecimentos sobre a vigilância em saúde $(22,2 \%)$. Em relação as fragilidades, os residentes relataram em maior porcentagem a leitura de muitos artigos em um único dia $(61,1 \%)$, dificuldade em interpretar artigos com temas diferentes da área de atuação $(22,2 \%)$ e muito tempo de vivência no SUS $(16,7 \%)$. Conclui-se que a metodologia ativa apresenta pontos positivos e negativos, como relatado pelos residentes, porém o modelo favorece uma forma de ensino-aprendizagem necessária à implementação dos residentes médicos veterinários para atuação no SUS.
\end{abstract}

Palavras-chave: residentes; Sistema Único de Saúde; metodologia; Saúde pública.

\begin{abstract}
The Residency Program in Occupational Health in Veterinary Medicine of the Federal Rural University of Pernambuco has the objective of training Veterinary Residents by means of in-service training for public health work in the areas of health surveillance. Thus, the present study aimed to describe the method used in the theoretical and practical training of residents, prior to their insertion into the Unified Health System (UHS), highlighting potentialities and weaknesses. Before the beginning of the activities in the Health Surveillance Secretariat and in the Family Health Support Center (FHSC), two courses were offered using active methodologies: Public Policies in Health and Integration Teaching Community Service. At the end of each course, approved residents (100\% of registered residents) were asked to report their opinions on them, highlighting potentialities and weaknesses. The most reported potentialities were: use of active methodologies such as Problem Based Learning (PBL) and case studies (77.8\%), reading of articles and writing of abstracts $(27.8 \%)$ and clarifications on health surveillance $(22.2 \%)$. In relation to fragilities, residents reported a high percentage of reading in a single day (61.1\%), difficulty in understanding papers from different fields than theirs $(22.2 \%)$ and a long time in UHS (16.7\%). It is concluded that the active methodology presents positive
\end{abstract}


and negative points, as reported by the residents, however the model favors a form of teaching-learning necessary for the implementation of veterinary medical residents in SUS.

Keywords: residents; Unified Health System; methodology; Public health.

\section{Introdução}

As funções do médico veterinário e a contribuição desse profissional na saúde humana são destacadas com a apresentação do cenário atual e as tendências para a Medicina Veterinária Preventiva e a Saúde Pública (Pfuetzenreiter et al., 2004). Desde 1998, pela Resolução do Conselho Nacional de Saúde (CNS) no 287/98, a Medicina Veterinária é reconhecida no Brasil como profissão da área de Saúde (BRASIL, 1998).

Recentemente, mais um passo para confirmar a atuação desse profissional na saúde pública foi sua inclusão na lista de profissionais que podem compor o Núcleo de Apoio à Saúde da Família (NASF), segundo Portaria do Ministério da Saúde/Gabinete do Ministro (MS/GM) n ${ }^{\circ} 2.488$, de 21 de outubro de 2011 (BRASIL, 2011).

De acordo com a lei orgânica 8080/90 (BRASIL, 1990), a formação de recursos humanos na área de saúde está incluída no campo de atuação do Serviço Único de Saúde (SUS). A portaria GM/MS n. 1.996/97 (BRASIL, 2007) reforçou o conceito de educação permanente como prática educativa, ancorada no trabalho e no conhecimento prévio dos trabalhadores, na problematização da realidade, na aprendizagem significativa e na transformação da prática, sugerindo que tais estratégias fossem construídas de maneira pactuada, com o envolvimento de atores tanto do setor saúde (gestores, trabalhadores e usuários) como da educação (gestores, instituições de ensino e estudantes de cursos da área da saúde).

Devido a essa demanda, o Ministério da Saúde criou uma política nacional de formação e desenvolvimento para o conjunto de profissionais da saúde, mediante a necessidade de formação de recursos humanos para atuação no SUS. A política engloba, dentre outros programas, as Residências Multiprofissionais (Cavalheiro e Guimarães, 2011).

O Programa de Residência em Área Profissional da Saúde em Medicina Veterinária da Universidade Federal Rural do Pernambuco (UFRPE) iniciou em 2015, apresentando-se na forma de pós-graduação Latu sensu, modalidade de treinamento em serviço, abrangendo conteúdos teóricos e práticos. O objetivo do programa é capacitar residentes Médicos Veterinários para atuarem tanto nas onze áreas de concentração do Hospital Veterinário, bem como na saúde pública, nas áreas de vigilância em saúde (PRPPG/UFRPE, 2018).

A duração do programa é de 24 meses, com carga horária mínima de 5.670 horas, sendo $4.620 \mathrm{~h}$ de carga horária prática no Hospital Veterinário, 90h de disciplinas teóricas e 960 horas de carga horária prática destinadas a vivência em Saúde Pública, com atividades desenvolvidas na Secretaria de Vigilância em Saúde e no NASF.

Nas atividades práticas nos serviços de Saúde Pública os residentes ficam sob responsabilidade de um preceptor, que tem a função de articular os conhecimentos da universidade e do trabalho, além de auxiliar na construção de soluções para possíveis problemas que surgirão na prática em saúde (Forte et al., 2015).

Pensando nas características dos profissionais que está sendo exigido no mercado, com perfil crítico-reflexivo e capazes de trabalhar em equipe, novas práticas foram adotadas na perspectiva de integrar ensino/serviço, teoriaprática, além de buscar desenvolver a capacidade de reflexão de problemas e elaboração de ações capazes de transformar a realidade social (Marin et al., 2010). Segundo Cavalheiro e Guimarães (2011), as universidades precisam ampliar e aprofundar as discussões sobre a formação profissional, particularmente na área da saúde.

Nesse contexto, o uso de metodologias ativas vem ganhando destaque na aprendizagem, podendo ser definida como as formas que os docentes utilizam para desenvolver o processo do aprender, na busca de conduzir a formação crítica de futuros profissionais (Borges e Alencar, 2014). Nessa perspectiva, o docente é denominado tutor, que é tido como um facilitador no processo ensinoaprendizagem, acreditando na capacidade do aluno de desenvolver e aprender (Mitre et al., 2008)

Considerando o uso de metodologias ativas como alternativa para aprendizagem dos profissionais de saúde que o Programa de Residência vem implementando na formação de 
médicos veterinários residentes para atuação no SUS, o presente estudo objetivou descrever a metodologia utilizada na formação teórico-prática dos residentes, previamente à inserção dos mesmos no Sistema Único de Saúde, destacando suas potencialidades e fragilidades.

\section{Material e Métodos}

Previamente ao início das atividades na Secretaria de Vigilância em Saúde e no NASF, foram oferecidas duas disciplinas aos residentes de medicina veterinária, sendo a primeira delas intitulada como "Políticas Públicas em Saúde", com carga horária total de 45 horas, cujo objetivo principal foi apresentar as principais políticas públicas em saúde e campos para atuação do médico veterinário no SUS.

Também foi objetivado o aprimoramento dos conhecimentos do residente nos campos de atuação da Vigilância em Saúde (vigilância epidemiologia, sanitária e ambiental), fornecendo assim subsídios para a atuação nas atividades práticas na Vigilância em Saúde, na atenção Básica, no diagnóstico situacional e na promoção de estratégias de ação no NASF.

Durante esta disciplina, foram abordados os seguintes conteúdos: Constituição Federal de 1998, Lei Orgânica da Saúde, Política Nacional de Controle de Zoonoses, Política Nacional de Educação Permanente em Saúde, Diretrizes para execução das ações de Vigilância em Saúde e Política Nacional de Atenção Básica. Os assuntos foram ministrados por meio de metodologia passiva com auxílio de slides expositivos, onde o docente é o protagonista da educação, e através da metodologia ativa, por meio da Aprendizagem Baseada em Problemas (ABP), estudos de casos e apresentação de filmes e curtas-metragens sobre as diferentes políticas públicas de saúde.

Ao final de cada aula, era apresentado aos residentes uma situação problema relacionada ao tema que seria abordado na próxima etapa, de forma que o conhecimento fosse buscado e gerado pelo próprio grupo envolvido na resolução da situação, praticando assim a aprendizagem entre times. Essas situações problemas eram resolvidas em grupo e posteriormente discutidas com toda a turma e o professor. Ao total, foram apresentadas quatro situações problemas que continham temas relativos a Diferenças entre Saúde Coletiva e Saúde Pública, Vigilância em Saúde com enfoques nas competências da vigilância epidemiológica, ambiental e sanitária, Atenção Básica com enfoque na formação das equipes de saúde da família e Diagnosticando a Situação de Saúde da Cidade Esperança.

A quarta e última situação problema, a Cidade Esperança, apresentava diversos problemas relacionados a saúde da população, como falta de saneamento básico, uso de drogas, gravidez indesejada, prostituição, lixo, entre outras. Nessa etapa, os residentes tiveram que apresentar soluções para os problemas através da implementação de ações já existentes no SUS relacionadas aos Estados e municípios, além de colocar em prática os conhecimentos adquiridos durante toda a disciplina.

A segunda disciplina ministrada foi "Integração Ensino/Serviço/Comunidade", de mesma carga horária, tendo como objetivo facilitar e proporcionar aos residentes um entendimento sobre a Integração Ensino/Serviço/Comunidade (IESC) para a vivência das atividades práticas no SUS. A disciplina esclareceu o papel do preceptor do serviço de saúde e a relação preceptor/residente enquanto atores na relação ensino/aprendizagem no cenário dos usuários do SUS, além de relatar experiências de outros programas que pudessem servir como estratégia na melhoria da qualidade da relação residente/preceptor durante a vivência no SUS.

Nesta disciplina foram abordados os seguintes temas: Formação de recursos humanos para o SUS, Avanços e desafios no sistema de Vigilância em Saúde no Brasil, Desafios e perspectivas da IESC, Estratégias de avaliação da relação residente/preceptor nas práticas de saúde, Visitas as Secretarias Municipais de Saúde para planejamento das atividades no SUS, Trabalho em equipe multiprofissional e multidisciplinar, Integração ensino/serviço nos processos de mudança na formação superior dos profissionais de saúde, além da realização de visitas as Secretarias Municipais de Saúde para o conhecimento e interação com os preceptores dos serviços locais de saúde.

Os conteúdos foram ministrados por meio da discussão de casos, apresentação de filmes e curtasmetragens sobre as diferentes formas de integração ensino/serviço encontradas na Política Nacional de Educação Permanente em Saúde (PNEPS) e leitura e discussão de 18 artigos científicos sobre a integração Ensino-Serviço e Políticas Públicas de Saúde, no último dia da segunda disciplina. O docente entregou um artigo para cada aluno, onde o mesmo deveria lê-lo e elaborar um resumo para 
ser entregue a todos os residentes no dia da discussão dos mesmos, de forma que todo o grupo tivesse acesso a todos os artigos, ampliando a sua capacidade de aprendizagem.

A avaliação dos residentes ocorreu por meio da observação da evolução individual durante as disciplinas, considerando o ritmo e demanda de cada indivíduo, através da produção de um portfólio individual e coletivo ao final da disciplina de Políticas Públicas em Saúde que contemplava todas as atividades desenvolvidas e conhecimentos adquiridos durante o curso, e pela elaboração de um artigo científico ao final da disciplina de Integração Ensino/Serviço/Comunidade, com relato de experiência ou tema escolhido pelo residente relacionado às atividades no SUS.

As duas disciplinas - Políticas Públicas de Saúde e Integração Ensino/Serviço/Comunidade, foram obrigatórias a todos os 18 médicos veterinários residentes do primeiro ano (R1), do programa de residência em área profissional da saúde da UFRPE. Ao final de cada disciplina foi solicitado aos residentes que relatassem suas opiniões sobre as mesmas, elencando os pontos positivos/potencialidades e os pontos negativos/fragilidades, sem identificação dos mesmos e que os relatos fossem depositados em um envelope, garantindo-se o anonimato e o sigilo de cada relato.

\section{Resultados e Discussão}

A Tabela 1 apresenta as potencialidades relatadas pelos residentes ao final das duas disciplinas. No total, 77,8\% (14/18) dos residentes relataram que a maior potencialidade foi a metodologia utilizada, a qual estimulou o aluno na busca do conhecimento e por tratar-se de uma metodologia ativa, fez com que os residentes pesquisassem mais sobre os assuntos ministrados e sobre os problemas explanados para maiores esclarecimentos durante o decorrer da disciplina. Tais resultados mostram que a metodologia ativa é mais eficaz no processo ensino-aprendizagem que a metodologia de ensino conservadora/tradicional, como afirmado por Cotta et al. (2012).

Tabela 1. Número e percentual de respostas relativas às potencialidades elencadas pelos médicos veterinários residentes em relação as disciplinas Políticas Públicas em Saúde e Integração/Ensino/Serviço/Comunidade.

\begin{tabular}{lcc}
\hline \multicolumn{1}{c}{ Potencialidades } & $\mathbf{N}^{\mathbf{0}}$ & $\mathbf{\%}$ \\
\hline Metodologia ativa (Aprendizagem Baseada em Problemas (ABP) e estudos de casos) & $14 / 18$ & 77,8 \\
estimula o aluno na busca do conhecimento & & 27,18 \\
Leitura de artigos e elaboração de resumos & $4 / 18$ & 22,2 \\
Vigilância e sua sistematização & $4 / 18$ & 22,2 \\
Conhecimento da possibilidade de interação com profissionais de outras áreas e & $3 / 18$ & 16,7 \\
atuação interdisciplinar & $3 / 18$ & 16,7 \\
Apresentação do SUS e seu contexto histórico & $3 / 18$ & 16,7 \\
Contato com uma nova área da medicina veterinária & $2 / 18$ & 11,1 \\
Professor altamente capacitado & $2 / 18$ \\
Apresentar pontos práticos antes da vivência & $1 / 18$ & 11,1 \\
Estimula o residente a enfrentar e aceitar novos desafios & $1 / 18$ \\
Incentiva o aluno a falar em público & $1 / 18$ \\
Nortear como o médico veterinário pode atuar na saúde pública & 5,5 \\
Saber trabalhar com o coletivo; interação com a comunidade & 5,5 \\
\hline
\end{tabular}

Nossos resultados também corroboram com o de Gomes et. al. (2010), onde os autores acreditam que a metodologia problematizadora, que foi utilizada como precedente à abordagem dos assuntos teóricos nas disciplinas relatadas neste estudo, amplia as possibilidades de construção do conhecimento e faz os alunos terem um olhar crítico sobre a realidade.

A discussão de problemas e tentativas de resolução faz parte da Metodologia da Problematização (MP), onde ocorre a observação da realidade, seu estudo e posterior busca de possíveis soluções para aplicações práticas. Segundo Vieira e Panúncio-Pinto (2015), essa metodologia tem como objetivos desencadear o pensamento reflexivo e mobilizar o potencial social e político do estudante.

Nessa metodologia, após o estudo de um problema, podem surgir outros desdobramentos sobre o tema, exigindo um contato com conteúdos que não foram expostos pelo professor num primeiro momento, mas que precisam ser estudados para compreensão do problema, estimulando os residentes na busca contínua pelo conhecimento e facilitando o processo de ensinoaprendizagem, contribuindo para uma formação mais sólida do residente que irá atuar nas práticas 
em saúde pública no SUS, após a realização das disciplinas teóricas.

Porém, também houve reclamações (Tabela 2) em relação a metodologia utilizada, onde $16,7 \%$ (3/18) dos residentes relataram que ficaram muito livres/ soltos durante a disciplina, pois a mesma não tinha uma linha gradativa de conhecimento. As informações eram distribuídas de forma difusa e os residentes relataram não estar preparados para o ensino baseado nessa metodologia, por ser diferente do modelo de ensino convencional, corroborando com estudos de Marin et al. (2010).

Sobre a leitura de textos e artigos científicos, com posterior elaboração do resumo, 27,8\% dos residentes ressaltaram que a leitura serviu para situá-los sobre possíveis atribuições durante a vivência, além de tratar de algumas problemáticas que eles poderiam enfrentar nas atividades práticas, enquanto 5,5\% não achou proveitosa a leitura dos artigos.

Por outro lado, esse mesmo ponto foi a fragilidade que apresentou maior queixa $(61,1 \%)$ entre os residentes, de acordo com os dados da Tabela 2. Onze residentes se queixaram do curto prazo para discussão dos artigos, causando o que foi relatado por um deles como "fadiga mental". Vieira e Panúncio-Pinto (2015) afirmam que essa metodologia requer a dedicação de mais tempo para o ensino, exigindo a disponibilidade do professor para pesquisar, acompanhar e cooperar no aprendizado do estudante, num processo compartilhado de construção do conhecimento.

Tabela 2. Número e percentual de respostas relativas às fragilidades elencadas pelos médicos veterinários residentes em relação as disciplinas Políticas Públicas em Saúde e Integração/Ensino/Serviço/Comunidade.

\begin{tabular}{lcc}
\multicolumn{1}{c}{ Fragilidades } & $\mathbf{N}^{\mathbf{o}}$ & $\mathbf{\%}$ \\
\hline Discussão de muitos artigos em um único dia, tornando-se cansativo & $11 / 18$ & 61,1 \\
Artigos com temas diferentes da área de atuação do residente, o que dificultou a & $4 / 18$ & 22,2 \\
interpretação & $3 / 18$ & 16,7 \\
Tempo muito extenso para exposição das ideias & $3 / 18$ & 16,7 \\
Tempo curto de disciplina e assunto muito extenso & $3 / 18$ & 16,7 \\
Muito tempo de vivência no SUS, tirando os residentes da rotina do hospital & $3 / 18$ & 16,7 \\
A metodologia deixa o aluno muito livre e alguns residentes não estavam & & \\
preparados para ter aula com essa metodologia & $2 / 18$ \\
Cidade esperança apresentada por muitos grupos, tornando-se repetitivo & $1 / 18$ \\
Discente muito rígido & $1 / 18$ & 11,1 \\
Não achou proveitosa a leitura dos artigos & 5,5 \\
\hline
\end{tabular}

Outra fragilidade relatada foi a dificuldade de interpretação dos artigos, pois os residentes atuam também em outras áreas da medicina veterinária e não tinham íntima relação com a saúde pública e os termos utilizados nesta área. Alguns residentes $(22,2 \%)$ relataram que os artigos não eram objetivos, eram muito extensos e não agregavam informações concretas. Esta dificuldade encontrada no ensino da PósGraduação, é reflexo do ensino da Graduação, como mostrado por Franco e Silva (2017), onde há uma realidade angustiante nos alunos de ensino superior. Estudos sobre o tema leitura apontaram que os discentes ingressam no meio universitário apresentando muitas dificuldades na leitura, e não conseguem compreender os textos solicitados pelos docentes (Franco e Silva, 2017).

Pereira et al. (2017) descreveram que os contextos escolares veem a leitura apenas como decodificação e não como um processo de interpretação e formação de ideias e opiniões próprias, porém este fato foi relatado por apenas um aluno (5,5\% dos residentes) como "fadiga mental".

Em relação ao conteúdo sobre as diretrizes para a execução das ações em Vigilância em Saúde e sua sistematização, o embasamento prévio, fornecido durante as duas disciplinas focos deste estudo, foi de fundamental importância para aprimorar o conhecimento teórico dos residentes sobre as competências das vigilâncias sanitária, ambiental e epidemiológica, para que, no momento do desenvolvimento da carga horária prática no serviço, soubessem o papel real de cada vigilância.

O outro ponto ressaltado foi o conhecimento sobre a possibilidade de execução de ações interdisciplinares $(22,2 \%)$, que é um dos mais importantes para que ocorra essa real integração Ensino/ Serviço/ Comunidade. Segundo Albuquerque et al. (2008) essa integração é de fundamental importância pois visa à qualidade de atenção à saúde individual e coletiva, a qualidade da formação profissional e a satisfação dos trabalhadores do serviço, ampliando as práticas e 
os saberes, tanto entre os profissionais, como entre os usuários do SUS.

De uma forma geral foi possível perceber que $16,7 \%$ dos residentes relataram ter gostado desse contato com uma "nova" área na medicina veterinária, área esta considerada como "nova" já que em muitas universidades esse tema foi pouco explorado pelos professores, além do que alguns residentes ainda relataram que nunca tiveram contato com a Saúde Pública durante a graduação. Entretanto, apesar do relato positivo, outros residentes $(16,7 \%)$ acharam que o tempo de vivência destinado às práticas de vivência no SUS seria muito extenso (960 horas), onde os residentes teriam que sair da rotina do hospital veterinário para as atividades na Vigilância em Saúde.

Também verificou-se neste estudo que $16,7 \%$ dos residentes teceram comentários positivos sobre o docente que ministrou as duas disciplinas, dizendo que o mesmo era altamente capacitado e atuante na área de saúde pública, relatando suas experiências no serviço. Durante as aulas, o docente procurou sempre explicitar sua vivência prática para melhor esclarecer e mediar o conteúdo em sala de aula, tornando a disciplina muito mais interessante devido a essa troca entre docente e aluno. Para Borges e Alencar (2014) o docente e seus recursos didáticos tem grande importância no aprendizado crítico-reflexivo dos seus alunos, de forma ativa e motivadora. Houve apenas uma queixa negativa quanto ao professor em relação a sua rigidez em sala de aula $(5,5 \%)$, exigindo muito dos residentes.

\section{Conclusão}

Uma vez definido pela legislação que o SUS é campo de prática para ensino e pesquisa, as instituições de ensino superior devem ser responsáveis em prover os serviços de saúde com formação de recursos humanos adequados, por meio do desenvolvimento de novas estratégias pedagógicas, sendo portanto, a identificação de potencialidades e fragilidades neste processo de formação para o SUS, fundamental para aprimorar mudanças no ensino e no serviço, promovendo a qualificação enquanto processo de trabalho no SUS e para o SUS, garantindo-se assim a integralidade da atenção ao usuário.

Conclui-se com este estudo que o uso de metodologias ativas permitiu a formação crítica e reflexiva dos residentes e que sua adoção enquanto estratégia de formação parece ser um grande desafio na formação superior dos profissionais da saúde. Ainda que a metodologia ativa apresente suas limitações e fragilidades, como relatado pelos residentes, o modelo favorece uma forma de ensino-aprendizagem necessária à implementação dos residentes médicos veterinários no SUS, além de representar um movimento inovador de educação na área da saúde, que muitas vezes está centrada no modelo tradicional da educação com aulas expositivas.

Os resultados obtidos neste trabalho servirão para o aprimoramento da ementa e da metodologia aplicada a estas disciplinas estudadas, de forma que as fragilidades sejam revistas pelo docente para que os discentes matriculados em semestres posteriores tenham um melhor aproveitamento.

\section{Conflito de Interesse}

Os autores declaram não existir conflito de interesse.

\section{Referências}

Albuquerque, V.S.; Gomes, A.P.; Rezende, C.H.A.; Sampaio, M.X.; Dias, O.V.; Lugarinho, R.M. Revista Brasileira de Educação Médica, 32(3): 356-362, 2008.

Brasil. Lei $\mathbf{n}^{\mathbf{0}}$ 8080, de 19 de setembro de 1990. Diário Oficial da União. Brasília, 20 set. 1990; Seção 1. Disponível em: <http://www.planalto.gov.br/ccivil_03/leis/ L8080.htm>. Acesso em: 20 jul. 2017.

Brasil (Ministério da Saúde). Resolução CNS No 287, de 8 de outubro de 1998. Disponível em:

<http://bvsms.saude.gov.br/bvs/saudelegis/c ns/1998/res0287_08_10_1998.html>.

Acesso em: 20 jul. 2017.

Brasil. Portaria n. 1.996 GM/MS, de 20 de agosto de 2007. Diário Oficial da União, Brasília, 22 ago. 2007. Seção 1, p. 34. Disponível em: <http://bvsms.saude.gov.br/bvs/saudelegis/ gm/2007/prt1996_20_08_2007.html>. Acesso em: 20 jul. 2017.

Borges, T.S.; Alencar, G. Metodologias ativas na promoção da formação crítica do estudante: o uso das metodologias ativas como recurso didático na formação crítica do estudante do ensino superior. Cairu em Revista, 3(4): 119-143, 2014.

Cavalheiro, M.T.P.; Guimarães, A.L. Formação para o SUS e os desafios da Integração Ensino Serviço. Caderno FNEPAS, 1(1): 19-27, 2011. 
Cotta, R.M.M.; Silva, L.S.; Lopes, L.L.; Gomes, K.O.; Cotta, F.M.; Lugarinho, R.; Mitre, S.M. Construção de portfólios coletivos em currículos tradicionais: uma proposta inovadora de ensino-aprendizagem. Ciência \& Saúde Coletiva, 17(3): 787-796, 2012.

Forte, F.D.S.; Pessoa, T.R.R.F.; Freitas, C.H.S.M.; Pereira, C.A.L.; Junior, P.M.C. Reorientação na formação de cirurgiõesdentistas: o olhar dos preceptores sobre estágios supervisionados no Sistema Único de Saúde (SUS). Interface - Comunicação, Saúde, Educação, 19(1): 831-843, 2015.

Franco, J.R.; Silva, N.V. Leitura: habilidade essencial no ensino superior. Disponível em:

<http://www.atenas.edu.br/faculdade/arquiv os/NucleoIniciacaoCiencia/RevistaCientific a/REVISTA\%20CIENTIFICA\%202010/14 $\%$ 20HABILIDADE\%20ESSENCIAL\%20 NO\%20ENSINO\%20SUPERIOR.pdf $>$.

Acesso em: 20 jul. 2017.

Gomes, A.P.; Arcuri, M.B.; Cristel, E.C.; Ribeiro, R.M.; Souza, L.M.B.M.; Siqueira-Batista, R. Avaliação no ensino médico: o papel do portfólio nos currículos baseados em Metodologias Ativas. Revista Brasileira de Educação Médica, 34(3): 390-396, 2010.

Marin, M.J.S.; Lima, E.F.G.; Paviotti, A.B.; Matsuyama, D.T.; Silva, L.K.D.; Gonzales, C.; Druzian, S.; Ilias, M. Aspectos das fortalezas e fragilidades no uso das Metodologias Ativas de aprendizagem. Revista Brasileira de Educação Médica, 34(1): 13-20, 2010.
Mitre, S.M.; Siqueira-Batista, R.; Girardi-deMendonça, J.M.; Morais-Pinto, N.M.; Meirelles, C.A.B.; Pinto-Porto, C.; Moreira, T.; Hoffmann, L.M.A. Metodologias ativas de ensino-aprendizagem na formação profissional em saúde: debates atuais. Ciência \& Saúde Coletiva, 13(2): 21332144, 2008.

Pereira, P.R.S.; Ataíde, M.B.C.; Carneiro, A.P.S. Leitor proficiente: utilização das estratégias de leitura em sala de aula. Disponível em: <http://editorarealize.com.br/revistas/fiped/t rabalhos/Trabalho_Comunicacao_oral_idin scrito_1278_f5cde87c549ad34dc98669b99 0025e67.pdf>. Acesso em: 20 jul. 2017.

Pfuetzenreiter, M.R.; Zylbersztajn, A.; Avila-Pires, F.D. Evolução histórica da medicina veterinária preventiva e saúde pública. Ciência Rural, 34(4): 1661-1668, 2004.

PRPPG UFRPE. Pró-Reitoria e Pesquisa e PósGraduação da Universidade Federal Rural de Pernambuco. Edital do processo seletivo para ingresso no Programa de Residência em Área Profissional de Saúde em Medicina Veterinária da Universidade Federal Rural de Pernambuco - 2018. Disponível em: <http://www.prppg.ufrpe.br/sites/www.prp pg.ufrpe.br/files/noticias/edital_residencia_ 2018.pdf>. Acesso em 14 mai. 2018.

Vieira, M.N.C.M.; Panúncio-Pinto, M.P.A. Metodologia da Problematização (MP) como estratégia de integração ensinoserviço em cursos de graduação na área da saúde. Medicina, 48(3): 241-248, 2015. 